\title{
Application Research for the Urban Ecological Index Prediction Based on the Grey System Prediction Model
}

\author{
$\mathrm{Li} \mathrm{Yu}^{1, *}$ and Jiaquan Wang ${ }^{2}$ \\ ${ }^{1}$ School of Computer Science and Project, Anhui University of Science and Technology, Huainan 232001, China \\ ${ }^{2}$ School of Resources and Environmental Engineering, Hefei University of Technology, Hefei 230009, China \\ ${ }^{*}$ Corresponding author
}

\begin{abstract}
City along the Huaihe river in understanding the natural environment and social economic conditions, based on statistics from the city's urban ecological support systems ecology quantitative 2006-2010 values, and accordingly based on multiple factors associated predictive model of gray system GM $(1, N)$ and the corresponding test methods, for 2011 to 2020 for urban ecosystem index for prediction. Good prediction is achieved.
\end{abstract}

Keywords-urban ecological indicators; gray model; forecast

\section{INTRODUCTION}

When we thought the application of operations research methods to solve practical problems, formulate development strategies and policies, decision-making on major issues must be scientific prediction of the future.

The prediction is based on development law of objective things of the past and present, by means of scientific methods to describe and analyze the future development trend and status, and hypothesis and scientific judgment are formatted. When the thought method we applied operations research to solve practical problems, formulate development strategies and policies, to make important decision, must carry on the scientific prediction on the future.

Grey system theory is to study and solve the gray system analysis, modeling, forecasting, decision-making and control theory. Grey prediction is made for gray system prediction, some of the commonly used prediction methods (such as regression analysis, etc.), you need a larger sample, if the sample is small, often cause large errors, the prediction target failure. Grey forecasting model modeling needed less information, convenient operation, high modeling precision, has a wide application in the field of all kinds of forecasting, is an effective tool for dealing with small sample prediction.

Grey system theory is composed of Huazhong University of science and Technology Professor Deng Julong proposed in 1982 and developed. More than twenty years, has aroused a lot of attention of scholars at home and abroad, has got great development. At present, in our country has become a social, economic, science and technology and many other areas of decision-making, prediction, evaluation, planning control, system analysis and modeling is one of the important ways. Especially the analysis and modeling of its short time series, statistical data, the less the incomplete information system, has the unique function, therefore has been applied widely in many fields [1 3].

\section{CONSTRUCTION OF THE GREy SyStem ModeL}

Make $x^{(0)}$ to the original sequence:

$$
x^{(0)}=\left[x^{(0)}(1), x^{(0)}(2), \ldots, x^{(0)}(n)\right]
$$

$$
x^{(1)}=\left[x^{(1)}(1), x^{(1)}(2), \ldots, x^{(1)}(n)\right]
$$

If $x^{(1)}$ and $x^{(0)}$ satisfy the following relationship between:

$$
\begin{gathered}
x^{(1)}(k)=\sum_{i=1}^{k} x^{(0)}(i) ; k=1,2, \ldots, n \\
x^{(r)}(k)=\sum_{i=1}^{k} x^{(r-1)}(i) \\
x^{(r)}(k)=\sum_{i=1}^{k-1} x^{(r-1)}(i)+x^{(r-1)}(k)=x^{(r-1)}(k-1)+x^{(r-1)}(k) \\
x^{(r)}(k)=\sum_{i=1}^{k} x^{(r-1)}(i)=\sum_{i=1}^{k}\left(\sum_{j=1}^{i} x^{(r-2)}(j)\right)
\end{gathered}
$$

Accumulation generation in the grey system theory has a very important position, it can make any nonnegative number column, swing or swinging, translate into a reduction, increasing sequence.

Based on the above mechanism, the following specific model and calculation formula of $G M(1,1)$. A non-negative original sequence. 


$$
X^{(0)}=\left\{x^{(0)}(1), x^{(0)}(2), \ldots, x^{(0)}(n)\right\}
$$

To $X^{(0)}$ make an accumulation, generation number listed as:

$$
x^{(1)}(k)=\sum_{i=0}^{k} x(i)
$$

So for albino forms of differential equations:

$$
\begin{gathered}
\frac{d x^{(1)}}{d t}+a x^{(1)}=u \\
x^{(0)}(k+1)=a\left[-\frac{1}{2}\left(x^{(1)}(k)+x(1)(k+1)\right)\right]+u
\end{gathered}
$$

The parameter vector least squares method to calculate available, namely:

$$
\hat{\Phi}=[\hat{a}, \hat{u}]^{T}=\left(B^{T} B\right)^{-1} B^{T} Y
$$

To calculate the parameters of the plug, and find out the discrete solution for:

$$
\begin{gathered}
\hat{x}^{(1)}(k+1)=\left[x^{(1)}(1)-\frac{\hat{u}}{\hat{a}}\right] e^{-\hat{a} k}+\frac{\hat{u}}{\hat{a}} \\
\hat{x}^{(0)}(k+1)=\hat{x}^{(1)}(k+1)-\hat{x}^{(1)}(k) \\
=\left(1-e^{\hat{a}}\right)\left[x^{(1)}(1)-\frac{\hat{u}}{\hat{a}}\right] e^{-\hat{a} k} \\
\frac{d x^{(1)}}{d t}+a x^{(1)}=u \\
\hat{U}=\left[\begin{array}{l}
\hat{a} \\
\hat{u}
\end{array}\right]=\left(B^{T} B\right)^{-1} B^{T} y \\
\hat{x}^{(1)}(k+1)=\left[x^{(1)}(1)-\frac{\hat{u}}{\hat{a}}\right] e^{-\hat{a} k}+\frac{\hat{u}}{\hat{a}}
\end{gathered}
$$

Associated factors is used in model $G M(1, N)$ to describe the dynamic development and $G M(1,1)$ is used to describe the independent factors, these independent factors commonly referred to as the dominant development system, related factors is called associated factors in the system.
System prediction is used the model to describe and predict the actual environment the common development of the various factors in the system. Suppose there are four factors $x_{1}, x_{2}, x_{3}, x_{4}$ in a system, $x_{2}$ influenced by factors $x_{1}, x_{2}$, which have dynamic association, you can establish a dynamic model to predict factors:

$$
\hat{x}^{(1)}(k+1)=\left[x^{(1)}(1)-\frac{\hat{u}}{\hat{a}}\right] e^{-\hat{a} k}+\frac{\hat{u}}{\hat{a}}
$$

$a_{21}, a_{22}, a_{23}$ is parameters of the system which can be gained through the model $G M(1,3)$.

If the $x_{3}$ affected $x_{2}$ factors of development, there is dynamic relation, the model can be established:

$$
d x_{3} / d t=a_{32} x_{2}-a_{33} x_{3}
$$

If the factors ${ }^{X_{1}}$ and ${ }^{X_{4}}$ is the independent development, establish the model respectively:

$$
\begin{aligned}
& d x_{1} / d t=a_{11} x_{1}+u_{1} \\
& d x_{4} / d t=a_{44} x_{4}+u_{4}
\end{aligned}
$$

Simultaneous equations above, below in the form of a matrix differential equations can be obtained, or the matrix equation is shorthand for:

$$
\left[\begin{array}{l}
d x_{1} / d t \\
d x_{2} / d t \\
d x_{3} / d t \\
d x_{4} / d t
\end{array}\right]_{=}\left[\begin{array}{cccc}
-a_{11} & 0 & 0 & 0 \\
a_{21} & -a_{22} & a_{23} & 0 \\
0 & a_{32} & -a_{33} & 0 \\
0 & 0 & 0 & -a_{44}
\end{array}\right]\left[\begin{array}{c}
x_{1} \\
x_{2} \\
x_{3} \\
x_{4}
\end{array}\right]_{+}\left[\begin{array}{c}
u_{1} \\
0 \\
0 \\
u_{4}
\end{array}\right]
$$

$$
\begin{gathered}
A=\left[\begin{array}{cccc}
-a_{11} & 0 & 0 & 0 \\
a_{21} & -a_{22} & a_{23} & 0 \\
0 & a_{32} & -a_{33} & 0 \\
0 & 0 & 0 & -a_{44}
\end{array}\right] \\
X=\left[\begin{array}{c}
x_{1} \\
x_{2} \\
x_{3} \\
x_{4}
\end{array}\right]
\end{gathered}
$$


The above differential equations, numerical solution can be obtained by using the runge-kutta method, and the development and changes of various factors to the system that can dynamically forecast.

\section{III. $G M(1, N)$ Prediction OF HuAiHe RIVER City ECOLOGICAL INDEX}

The existing number of population in some areas, food consumption, farming, food is average mu produce etc. Several reference values are shown as Table 1 (2006-2011), this paper used $G M(1, N)$ model for their establishment of forecasting system, implemented in MATLAB.

TABLE I. A REGION'S POPULATION, FOOD CONSUMPTION CHANGES, THE OUTPUT VALUE OF ANIMAL HUSBANDRY, THE AVERAGE PER MU YIELD OF GRAIN

\begin{tabular}{|c|c|c|c|c|c|c|}
\hline Year & $\mathbf{2 0 0 6}$ & $\mathbf{2 0 0 7}$ & $\mathbf{2 0 0 8}$ & $\mathbf{2 0 0 9}$ & $\mathbf{2 0 1 0}$ & $\mathbf{2 0 1 1}$ \\
\hline Population & 168561 & 172036 & 171226 & 172808 & 175562 & 182378 \\
\hline Grain & 156760 & 155172 & 162546 & 138986 & 155365 & 162260 \\
\hline Breeding & 506 & 656 & 736 & 618 & 602 & 1036 \\
\hline $\begin{array}{c}\text { Grain per } \\
\text { mu }\end{array}$ & 902 & 1136 & 1138 & 1006 & 1100 & 1162 \\
\hline
\end{tabular}

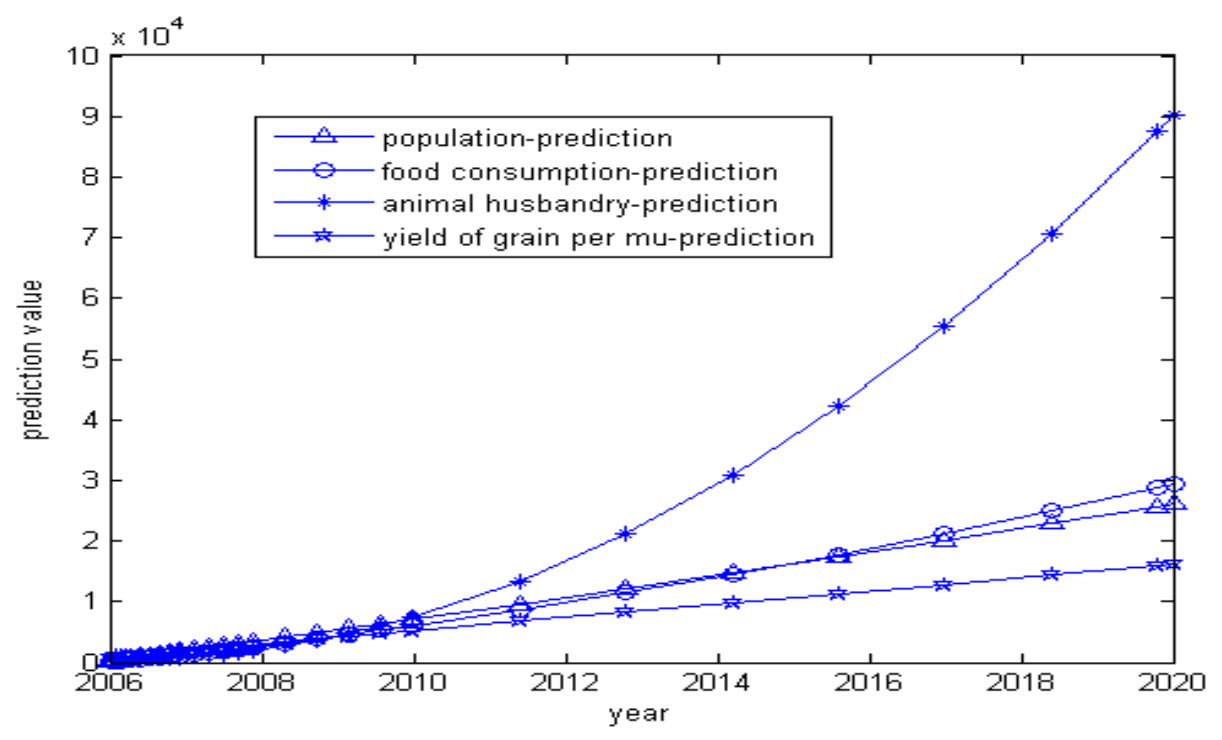

FIGURE I.

PREDICTION RESULTS OF THE GREY MODEL SYSTEM.

To calculate the parameters of the model, build a system of differential equations and to solve them, to predict the results. MATLAB program is used for programming.

\section{CONCLUSIONS}

Via behavior characteristic indexes of the system to establish a set of interrelated grey prediction model, predict numerous coordinated relationship between the variables in the system change. In this paper, an instance verify the feasibility of the method.
Estimate the system prediction model coefficient coefficient of differential equations.

\begin{tabular}{|c|c|c|c|}
\hline \multicolumn{4}{|l|}{$\mathrm{AA}=$} \\
\hline 0.0144 & 0 & 0 & 0 \\
\hline 2.2388 & -2.6755 & 0.2557 & 0 \\
\hline 0 & 0.7016 & -0.0852 & 0 \\
\hline 0 & 0 & 0 & -0.0013 \\
\hline \multicolumn{4}{|l|}{$\mathrm{UU}=$} \\
\hline \multicolumn{4}{|c|}{$1.0 \mathrm{e}+003 *$} \\
\hline \multicolumn{4}{|l|}{1.6613} \\
\hline \multicolumn{4}{|l|}{0} \\
\hline 0 & & & \\
\hline
\end{tabular}

1.1036

Prediction results of the grey model system are shown as Figure.1. In this problem each index numerical difference is bigger, in order to avoid build equations of differential equations become rigid and difficult to solve, to properly deal with the data, the population and grain consumption both numerical index down 100 times, namely, divided by 100, it is evident in the following program. 
[2] Luo Dang, Liu SiFeng, and Dang YiGuo, "The optimization of grey model GM(1,1),” China engineering science, 2003, 5(8), pp. 50-53.

[3] Wang zheng Xin, Dang Yao Guo, and Liu Si Feng, "An optimal GM(1,1) based on the discrete function with exponential law," Systems Engineering-Theory \& Practice, 2008, 28(2), pp. 61-67.

[4] Dang Y G, Liu S F, “The GM models that x(n) be taken as initial value,” Kybernetes, 2004, 33(2), pp. 247-255.

[5] Tsaur R C, "Forecasting analysis by using fuzzy grey regression model for solving limited time series data," Soft Computing, 2008, 12(11), pp. 1105-1113.

[6] Zeng Bo, “Approximate inhomogeneous exponential sequence at DGM $(1,1)$ direct modeling method. The system engineering theory and practice,” 2011, 31(2), pp. 297-301.

[7] Liu Si Feng, "Grey system theory and its applications," 2010(5), pp. 156-158, 227. 